Check for updates

Cite this: J. Mater. Chem. A, 2020, 8 17399

Received 17th July 2020

Accepted 11th August 2020

DOI: $10.1039 / \mathrm{d} 0 t a 06985 j$

rsc.li/materials-a

\section{Characterization of mechanical degradation in an all-solid-state battery cathode $\uparrow$}

\author{
Tan Shi, (D) t $^{\mathrm{ab}}$ Ya-Qian Zhang, (D) t $^{\mathrm{ac}}$ Qingsong Tu, ${ }^{a}$ Yuhao Wang, ${ }^{\mathrm{a}}$ M. C. Scott ${ }^{\star a c}$ \\ and Gerbrand Ceder ${ }^{\star a b}$
}

\begin{abstract}
Solid-state batteries (SSBs) are considered promising next-generation energy storage devices but tend to suffer from rapid capacity fade. Here, we demonstrate that mechanical contact loss between the solid conductor and cathode, induced by its volume changes during cycling, plays a significant role in the observed capacity fade. Focused ion beam-scanning electron microscope (FIB-SEM) tomography with nanoscale resolution was used for 3D characterization of the composite electrode morphology before and after cycling. The tomography data demonstrates the development of voids and cracks near the cathode particles and significant contact loss between the cathode particles and solid electrolyte after cycling. The observed mechanical degradation in the electrode composite highlights the difficulty and importance of engineering mechanically stable SSBs. The application of large external pressure after long-term cycling led to recovery of lost capacity and reduced the cell resistance, confirming the effect of mechanical degradation.
\end{abstract}

Solid-state batteries (SSBs) are of great interest for energy storage as they would improve safety compared with Li-ion batteries by replacing the flammable liquid electrolyte with an inorganic solid electrolyte. Potential energy density enhancements are also possible as the solid-state electrolyte may enable the use of Li metal. ${ }^{1,2}$ However, current SSBs often exhibit more rapid capacity fade than conventional Li-ion batteries. ${ }^{3-8}$ Improving the cyclability of SSBs requires a more comprehensive understanding of the degradation mechanism during cycling.

\footnotetext{
${ }^{a}$ Department of Materials Science and Engineering, University of California, Berkeley, CA 94720,USA.E-mail: gceder@berkeley.edu; mary.scott@berkeley.edu

${ }^{b}$ Materials Sciences Division, Lawrence Berkeley National Laboratory, Berkeley, CA 94720, USA

'The Molecular Foundry, Lawrence Berkeley National Laboratory, Berkeley, CA 94720, USA

$\dagger$ Electronic supplementary information (ESI) available. See DOI: 10.1039/d0ta06985j

¥ Co-authorship.
}

Both chemical and mechanical instabilities play a role in the impedance build-up and capacity fade during SSB cycling. Chemical reactivity issues between conductor and cathode have been well-studied theoretically ${ }^{9,10}$ and experimentally. ${ }^{5,11-13}$ In this work, we focus on the mechanical failure modes of an SSB composite cathode. The latter consists of an intimate mixture of the solid electrolyte and active cathode particles so that a transport path exists for the Li ions to/from the cathode particles. Good Li-ion transport across the solid electrolyte/ cathode particle interface is necessary to minimize the cell resistance and overpotential of the composite cathode. Chemical reactivity at the solid electrolyte/cathode interface can lead to the formation of a reaction layer, which increases cathode impedance. For example, sulfide-based electrolytes have been shown to react with oxide-based cathodes, resulting in overpotential growth and capacity fade..$^{5,9,11-13}$ Coating the cathode particles has been shown to mitigate this issue to some extent and improve cycling stability. ${ }^{14-18}$

Mechanical issues at the solid electrolyte/cathode interface are less studied than the chemical instability but may also cause significant capacity fade. The challenge to retain mechanical contact in the cathode composite mainly arises from the volume change of the cathode materials during cycling. Volume changes of up to $8 \%$ during cycling are possible for common layered cathode compounds. ${ }^{19,20}$ Because the elastic deformation of a solid electrolyte is extremely small, ${ }^{21}$ the only way that the solid electrolyte can accommodate the volume change of the cathode particles is through displacement or irreversible deformation (plastic deformation, cracking, etc.) of particles in the composite. Repeated expansion/contraction of the cathode particles as they charge and discharge is thus expected to lead to contact loss at the solid electrolyte/cathode interface and hinder Li-ion transport. ${ }^{22,23}$ Although there have been some reports discussing the mechanical degradation in SSB cathode composites, ${ }^{8,24,25}$ the evolution of the electrode microstructure and the severity of the contact loss during cycling remain unclear and require high-resolution 3D characterization and quantitative analysis of the cycled composite cathode. 
In this work, we use focused ion beam-scanning electron microscope (FIB-SEM)-based tomography with sub-100 nm spatial resolution to visualize and quantify the loss of mechanical contact in an SSB cathode composite after cycling. Quantitative analysis of the microstructural evolution and interface separation reveals a correlation between the capacity fade and the loss of physical contact between the cathode particles and solid electrolyte upon cycling.

In the current study, we investigate composite cathodes that combine a NMC cathode $\left(\mathrm{LiNi}_{0.5} \mathrm{Mn}_{0.3} \mathrm{Co}_{0.2} \mathrm{O}_{2}\right)$ with amorphous $75 \mathrm{Li}_{2} \mathrm{~S}-25 \mathrm{P}_{2} \mathrm{~S}_{5}$ (LPS) solid electrolyte. The cathode material was coated with an amorphous Li-Zr-oxide (LZO) layer to reduce the reaction between NMC and LPS and to minimize the effect of the chemical instability on the electrochemical performance. ${ }^{18}$ SSB cells were constructed with In metal as the anode, a composite of NMC/LPS/carbon nanofiber (CNF) in a weight ratio of $60: 35: 5$ as the cathode, and LPS as the solid electrolyte. All the cells were cycled between 1.4 and $3.7 \mathrm{~V}$ vs. In (between 2 and $4.3 \mathrm{~V} v$ s. $\mathrm{Li} / \mathrm{Li}^{+}$) using constant-current constantvoltage (CCCV) charging and constant-current discharging at a rate of $0.05 \mathrm{~mA} \mathrm{~cm}^{-2}$. The details of the cell fabrication have been previously reported and are provided in the ESI. $\dagger^{26}$

Fig. 1(a) presents the discharge capacity retention of the SSB. The first cycle shows a discharge capacity of $129 \mathrm{~mA} \mathrm{~h} \mathrm{~g}^{-1}$. Over the next 26 cycles, the discharge capacity gradually decreased to $102 \mathrm{~mA} \mathrm{~h} \mathrm{~g}{ }^{-1}$, corresponding to a loss rate of approximately $1 \mathrm{~mA} \mathrm{~h} \mathrm{\textrm {g } ^ { - 1 }}$ per cycle, as represented by the yellow line in Fig. 1(a). However, a rapid capacity decline occurred between the $30^{\text {th }}$ and $40^{\text {th }}$ cycle, with the cell capacity ending at less than $20 \mathrm{~mA} \mathrm{~h} \mathrm{~g}^{-1}$. Similar cycling behavior was consistently observed in other cells, although the sudden drop in capacity could be found earlier or later. The cycling performance of an additional (a)

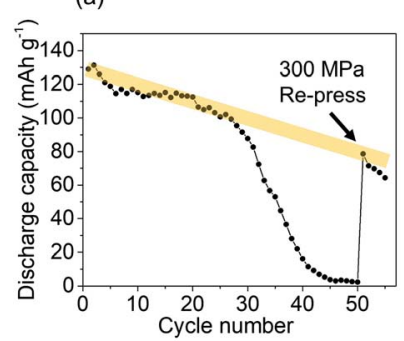

(c)

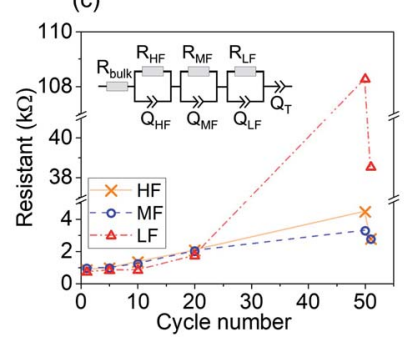

(b)

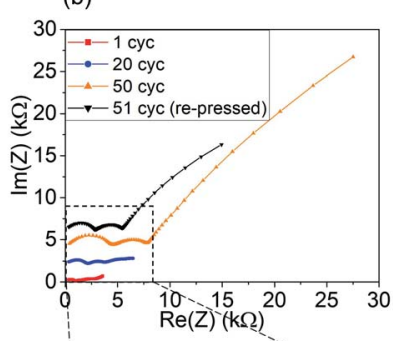

(d)

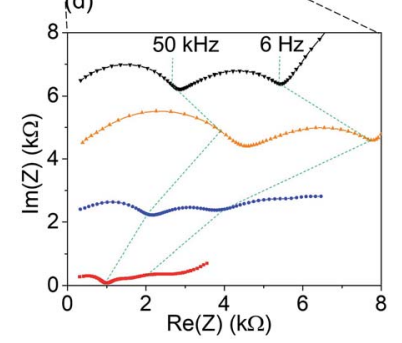

Fig. 1 Electrochemical tests of SSB before and after re-pressing. (a) Discharge capacity of the cell. Pressure of $300 \mathrm{MPa}$ was applied after 50 cycles. (b) and (d) EIS measurement after 1, 20, 50, and 51 cycles. (c) Summary of cell resistances determined through EIS measurements. The equivalent circuit is shown in the inset. cell with a large capacity drop after $\sim 30$ cycles is shown in Fig. S1.†

To investigate the mechanical contribution to this rapid capacity degradation, $300 \mathrm{MPa}$ pressure was reapplied to the cell after 50 cycles to restore the mechanical contact. Remarkably, after re-pressing the cell at this pressure, the discharge capacity of the SSB recovered to $\sim 80 \mathrm{~mA} \mathrm{~h} \mathrm{~g}{ }^{-1}$. This result unambiguously confirms that mechanical degradation significantly contributes to capacity loss during SSB cycling. In addition, the discharge capacity of $80 \mathrm{~mA} \mathrm{~h} \mathrm{~g}^{-1}$ after the re-pressing corresponds to an average capacity decay rate of $1 \mathrm{~mA} \mathrm{~h} \mathrm{~g}{ }^{-1}$ per cycle, which is consistent with the initial decay rate for the first 25 cycles. This finding suggests that there are likely two distinct degradation modes during SSB cycling: one that leads to gradual capacity decay at a relatively constant rate, which may be related to the chemical degradation, and another that leads to rapid capacity decay over several cycles, which can be attributed to the mechanical degradation.

Any contact loss in the composite cathode would lead to an increase in the interfacial resistance, which can be probed by electrochemical impedance spectroscopy (EIS). Fig. 1(b) and (c) present the EIS measurements of the cell after 1, 20, 50, and 51 (after re-pressing) cycles. Each spectrum consists of three distinct semicircles in the high-, mid-, and low-frequency regions. The different frequency regions originate from the different time constants for the charged species transport at various interfaces. The EIS results were fitted with the equivalent circuit model, and the cell resistances at different frequencies are summarized in Fig. 1(d). During the first 50 cycles, the resistance in all the frequency regions increased. After re-pressing the cell ( $51^{\text {st }}$ cycle), a significant decrease of all the resistances was observed. This general trend suggests that mechanical degradation is not limited to the cathode/solid electrolyte interface but may also occur at other interfaces such as the anode/solid electrolyte interface and solid electrolyte grain boundaries. Although it is not possible to precisely assign a single frequency region to the cathode/solid electrolyte interface because of the overlapping time constants of different transport processes, previous studies have argued that the midfrequency region is most sensitive to changes at the cathode/ solid electrolyte interface. ${ }^{7}$ Interestingly, the resistance corresponding to the mid-frequency region $\left(R_{\mathrm{MF}}\right)$ only increased from 954 to $1241 \Omega$ in the first 10 cycles, a $30 \%$ increase. After 50 cycles, it further increased to $3283 \Omega$, a $244 \%$ increase. This finding again demonstrates that interfacial degradation is accelerated in the later cycles, which is consistent with the observed faster capacity decay. After re-pressing, $R_{\mathrm{MF}}$ decreased to $2755 \Omega$, suggesting that the application of large external pressure results in the recovery of some contact area between the cathode and solid electrolyte and reduces the cell resistance. However, this value is still much larger than the original $R_{\mathrm{MF}}$ after the first cycle, suggesting that other factors, such as chemical degradation at the interfaces, also play a role in the resistance growth, or that not all contact can be restored. We note that it has become clear recently that LZO does not fully protect $\mathrm{NMC}$ at the charging voltage required for $\mathrm{NMC}^{27}$ and the 
observed capacity fade can be partially attributed to the instability of the LZO coating.

To directly observe and verify the possible contact loss between the cathode and solid electrolyte, FIB-SEM tomography was used to characterize the microstructural evolution in the composite cathode during cycling. Three SSB cells (before cycling, after 10 cycles, and after 50 cycles) were extracted in the discharged state and evaluated using FIB-SEM tomography. The workflow for the FIB-SEM tomography experiment is presented in Fig. 2. The SSB full cell was tilted at a $52^{\circ}$ angle with the cathode composite perpendicular to the ion beam. The ion beam was first used to mill trenches surrounding the area of interest, exposing the cross-section to the top-mounted electron beam at a $52^{\circ}$ angle. A backscattered electron (BSE) image was acquired to provide 2D morphology information on the cathode composite. Then, a thin slice $(50 \mathrm{~nm})$ was milled away from the cross-section with the ion beam, and another BSE image was taken. After repeating this process several hundred times, the image stack was aligned, cropped, and combined into a $2 \mathrm{D}$ image stack (Fig. 2(c)) (see ESI Fig. S2 and S3† for the detailed image process procedures). The $2 \mathrm{D}$ image stack and corresponding four-phase segmentation image are presented in Fig. 2(c) and (d), respectively, whereby the NMC cathode (blue), LPS solid electrolyte (yellow), carbon (black), and void (red) can be accurately distinguished. The segmented image stacks were then reconstructed into a $3 \mathrm{D}$ volume. The reconstructed structures of the composite cathodes before and after electrochemical cycling are presented in Fig. 3.

The segmented tomography data presented in Fig. 3 enables a quantitative analysis of the void development in the cathode composite during cycling. In the pristine sample, the voids only account for less than $3 \%$ of the total volume. Although the voids can be observed throughout the solid-electrolyte volume, they are mostly small and isolated. These small voids are formed during the cold-pressing process, when the solid-electrolyte particles undergo large deformation, leaving small voids at particle boundaries. After 10 cycles, the void morphology has not changed significantly, and the total void volume percentage increased by less than $0.5 \%$. This result suggests that in the first 10 cycles, during which only gradual capacity fade occurs, the mechanical degradation in the composite electrode is minimal or is not at the scale where it is visible or influences performance. A substantial change in the void morphology is observable after 50 cycles. The total void volume increased to $9.50 \%$ of the total volume, more than three times that in the pristine sample. The voids are also more connected, forming large flake-like cracks on the scale of $\sim 10 \mu \mathrm{m}$ near the cathode particles. To more carefully examine the void morphology, representative cross-sectional SEM images were acquired from each sample and are presented in Fig. 4(a)-(c). Although only small isolated pores are present in the samples before cycling and after 10 cycles, large cracks can be observed next to cathode particles after 50 cycles (highlighted by the red arrows). This location suggests that the repeated volume change of cathode particles during cycling is likely the main driving force for the void formation. Such voids near the surface of the cathode particles will directly hinder the Li transfer between the cathode and solid electrolyte, explaining the increase in the overpotential and capacity fade observed.

To better quantify the degree of contact loss at the cathode/ solid electrolyte interface in the sample that was cycled 50 times, we divided the cathode surface into cathode surface in contact with voids (contact loss) and cathode surface in contact with other components such as other cathode, solid electrolyte, or carbon (with contact), based on the segmentation shown in Fig. 3. Fig. 4(d) demonstrates the labeling of the two categories in a 2D slice of Fig. 4(c) with the contact loss surfaces highlighted in red, and the other cathode surfaces shown in blue. This analysis was performed on all images of the sample, and the reconstructed 3D structure of the cathode surfaces with (a)

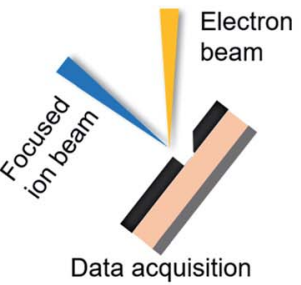

(c)

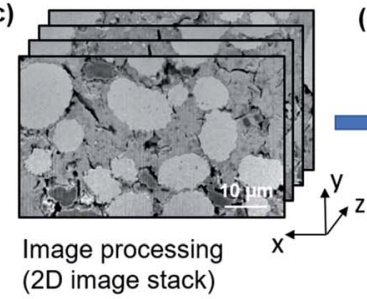

(b)

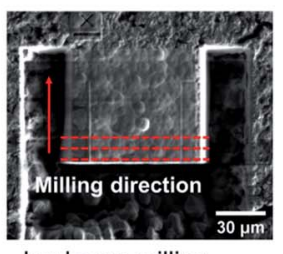

Ion beam milling

(d)

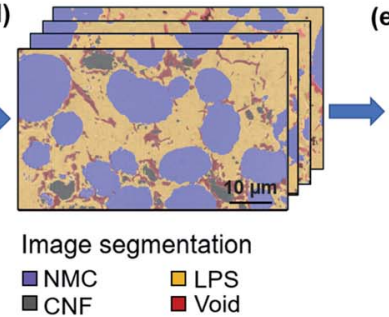

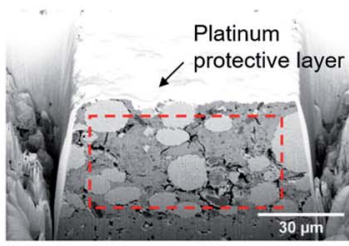

Electron beam Imaging

(e)

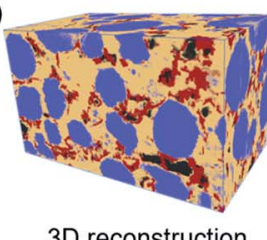

$3 \mathrm{D}$ reconstruction

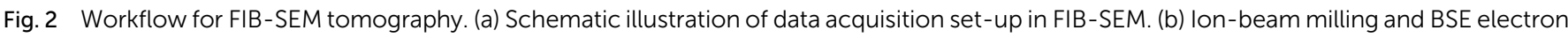

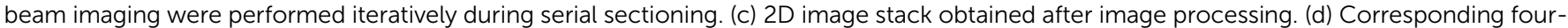

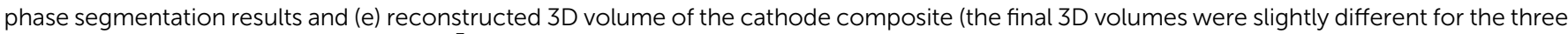
samples but were all $\sim 60 \times 40 \times 30 \mu \mathrm{m}^{3}$ ). 


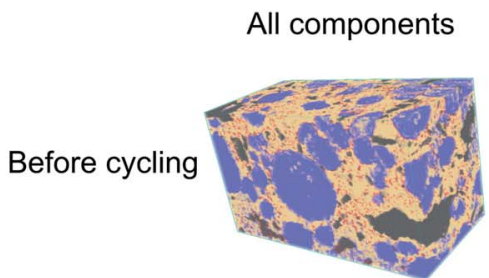

$\left(57 \times 33 \times 29 \mu \mathrm{m}^{3}\right)$

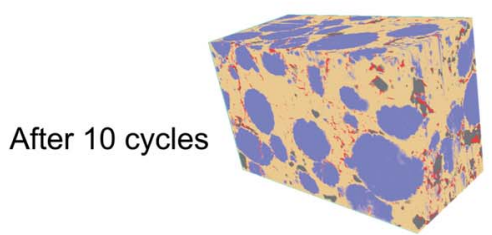

$\left(66 \times 41 \times 31 \mu \mathrm{m}^{3}\right)$

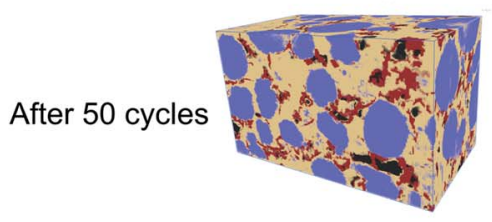

$\left(64 \times 38 \times 36 \mu \mathrm{m}^{3}\right)$
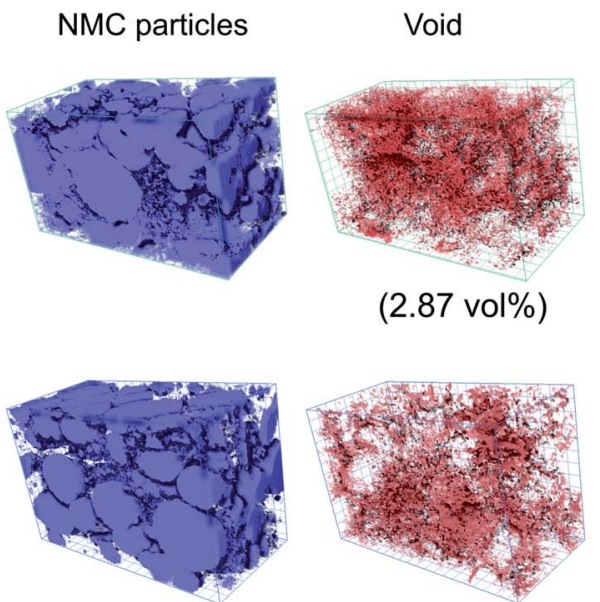

(3.23 vol\%)
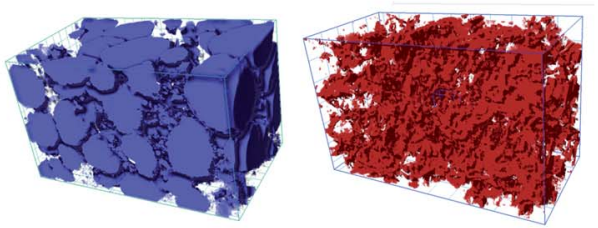

(9.50 vol\%)

Fig. 3 Reconstructed 3D structures of cathode composite. The figures correspond to the samples before cycling (top), after 10 cycles (middle), and after 50 cycles (bottom). The left column shows all the components (blue: NMC; yellow: LPS; red: void; black: carbon). The middle column shows only the NMC particles, and the right column shows only the voids. The overall volume and void volume percent are labeled below the figures.

contact (blue) and without contact (red) is presented in Fig. 4(e). Contact loss was observed at most of the cathode particle surfaces, demonstrating the severity of the mechanical problems caused by cathode volume change. The estimated contact loss area is $10.4 \%$ of the total cathode surface area.

To better visualize the local contact loss distribution around the cathode particles, a single NMC particle and its surrounding voids in the sample cycled 50 times were isolated from the larger volume and are shown in Fig. 4(f). The spherical NMC particle at the center is shown in grey, and the surrounding voids are shown in red. The voids are much more concentrated on the right side of the NMC particle, suggesting that the contact loss areas caused by the cathode particle volume shrinking will mostly be on one side of the particle, rather than uniformly distributed around the particle. This is expected because the presence of voids on one side of the particle is sufficient to release the strain caused by the particle volume change. This concentration of the decohered area in the cathode particle may aggravate its effect on capacity loss as now all the Li ions going in and out of the area under the contact loss have a much larger distance to travel before they reach the solid electrolyte. This is in contrast to if the contact loss area were homogeneously dispersed across the cathode particle. In the latter case, diffusion gradients parallel to the surface, resulting from blocked-out surface area for Li to enter or leave the particle, would rapidly fade out in the cathode particle.

The FIB-SEM tomography and electrochemical testing results together strongly suggest that the severe mechanical degradation is the main cause of the rapid capacity fade in the SSB composite electrode, particularly in later cycles. In current study, the crack development is mostly observed near the cathode/solid electrolyte interface, which suggests that the cathode particle volume change is the origin of the mechanical failure during cycling. Thus, minimizing this mechanical degradation is critical to extending the cycle life of SSBs.

It should be noted that the observed cathode/solid electrolyte delamination is likely only part of the overall mechanical degradation during SSB operation. Contact loss during cycling is also likely to occur at the anode side. The low-frequency region in the EIS data (Fig. 1), which is often attributed to the anode/solid electrolyte interface, ${ }^{7}$ sees a significant increase during cycling and decreases after pressing. This suggests some anode delamination could also exist and requires further study of the anode morphology. Moreover, although significant cathode particle cracking is not observed in the current study (Fig. 4(d)), it has often been observed in liquid cells, ${ }^{28,29}$ and could occur in SSBs with longer cycling and larger depth of charge and discharge.

Several routes can be envisaged to improve the mechanical response of the composite cathode, none of which is likely to be very easy. This includes the optimization of cathode morphology, cathode chemistry/structure, mechanical properties of the solid electrolyte, and use of external pressure. Reducing the cathode surface displacement is critical for lowering the mechanical strain during cycling, which can be 
(a) Before cycling

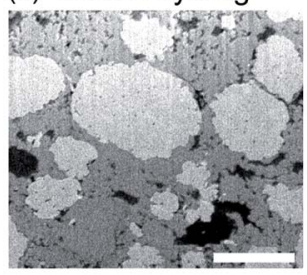

(c) 50 cycles

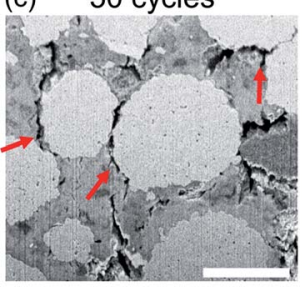

(e)

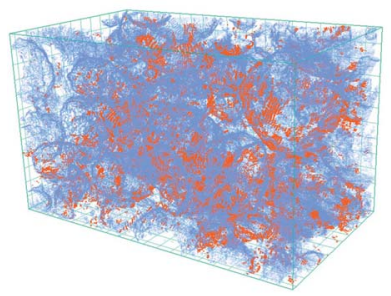

(b) 10 cycles

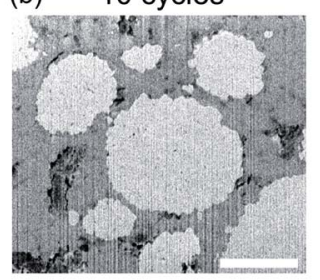

(d)

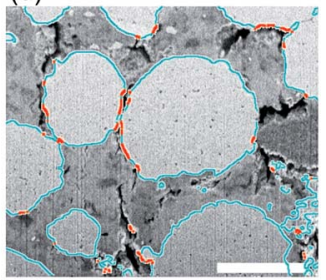

(f)

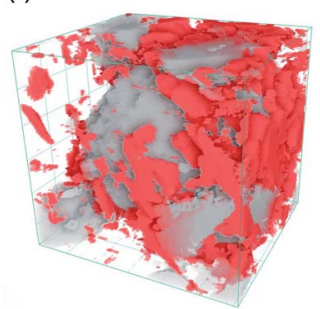

Fig. 4 Cross-sectional SEM images of composite cathode (a) before cycling, (b) after 10 cycles, and (c) after 50 cycles. Scale bar: $10 \mu \mathrm{m}$ (d) NMC surfaces (blue) with void/NMC interfaces highlighted in red and (e) their 3D reconstruction. (f) Part of the 3D reconstruction of the after-50-cycles sample showing an isolated NMC particle (grey) with surrounding voids (red). Other components (LPS and carbon) are not shown. Box sizes for (e) and (f) are $64 \times 38 \times 36 \mu \mathrm{m}^{3}$ and $18 \times 20 \times 18$ $\mu \mathrm{m}^{3}$, respectively.

achieved by decreasing the cathode particle size or cathode strain. For a cathode material with a specific strain, a smaller particle size would result in less surface displacement and therefore reduced stress at the cathode/solid electrolyte interface. However, reducing the cathode particle size could negatively affect the cathode utilization and energy density of SSBs. ${ }^{26}$ Reducing the cathode strain during cycling is another way to minimize mechanical issues. Different cathode chemistries and structures have been shown to lead to different volume change during cycling. ${ }^{19,30,31}$ The current results motivate the search for high-energy-density electrode materials with small volume change or "zero-strain". Different solid-electrolyte materials will also result in different mechanical degradation behavior. For example, the rapid development of cracks in the first few cycles was observed in SSBs with an oxide-based solid electrolyte, which is more rigid than the sulfide solid electrolyte used in the current study. ${ }^{25}$ In contrast, polymer-based composite solid electrolytes can accommodate larger strain and have been shown to improve the cycling stability. ${ }^{32-34}$ Therefore, developing a solid electrolyte with higher elasticity is an additional route to mitigate mechanical instability. Finally, applying a large external pressure (stack pressure) during cycling could in principle reduce the contact loss at the cathode/solid electrolyte interface, but such a large stack pressure may not be practical for large format cells.

It should be noted that the reason why the mechanical degradation and associated capacity fade accelerates in the later cycles remains unclear. One possible reason for this acceleration is that continuous chemical degradation conspires with the mechanical issues at the interface to accelerate the decohesion. Severe chemical reactions at the cathode/solid electrolyte interface have been widely reported ${ }^{5,8,12,13}$ and are likely to continue near the interface of LPS and the LZO-coated NMC upon cycling. ${ }^{27}$ The gradual growth of an interfacial layer composed of various reaction products can weaken the mechanical adhesion between the cathode and solid electrolyte, promoting crack development under stress. Or it may even lead to residual stresses which add to the stresses caused by the volume change of the cathode.

Another possibility is that in the early cycles, only very small microfractures occur, undetectable by the tomography and that these coalesce or lead to larger-scale decohesion in the later stage as is not uncommon for ductile fracture in materials.

\section{Conclusions}

In summary, using FIB-SEM tomography, we characterized SSB composite cathodes and revealed severe mechanical degradation during cycling. A large void volume $(9.50 \%)$ was observed in the cathode composite after 50 cycles. These voids were mostly distributed near the cathode particle surfaces, leading to a contact area loss of more than $10 \%$. The observed rapid capacity drop in later cycles is attributed to this mechanical contact loss. Our results also suggest, somewhat surprisingly, that mechanical degradation does not progress gradually starting from the first cycle. Instead, the majority of the void formation and contact loss likely occurs at later cycles and over a short period. The severe mechanical degradation observed in this study highlights the importance of mechanical considerations in SSB design.

\section{Conflicts of interest}

There are no conflicts to declare.

\section{Acknowledgements}

This work was supported by the Assistant Secretary for Energy Efficiency and Renewable Energy, Vehicle Technologies Office, of the U.S. Department of Energy under Contract No. DE-AC0205CH11231, under the Advanced Battery Materials Research (BMR) Program. We acknowledge support by the Samsung Advanced Institute of Technology. The FIB-SEM experiments were performed at the Molecular Foundry, LBNL. Work at the Molecular Foundry was supported by the Office of Science, Office of Basic Energy Sciences, of the U.S. Department of Energy under Contract No. DE-AC02-05CH11231. The authors also gratefully acknowledge Dr Taku Watanabe, Dr Yuichi Aihara, Dr Tomoyuki Tsujimura, and the Samsung R\&D Institute of Japan for providing the LZO-coated cathode materials. 


\section{References}

1 J. Janek and W. G. Zeier, Nat. Energy, 2016, 1, 16141.

2 J. Liu, Z. Bao, Y. Cui, E. J. Dufek, J. B. Goodenough, P. Khalifah, Q. Li, B. Y. Liaw, P. Liu, A. Manthiram, Y. S. Meng, V. R. Subramanian, M. F. Toney, V. V. Viswanathan, M. S. Whittingham, J. Xiao, W. Xu, J. Yang, X. Q. Yang and J. G. Zhang, Nat. Energy, 2019, 4, 180-186.

3 T. Ohtomo, A. Hayashi, M. Tatsumisago, Y. Tsuchida, S. Hama and K. Kawamoto, J. Power Sources, 2013, 233, 231-235.

4 Y. Kato, S. Hori, T. Saito, K. Suzuki, M. Hirayama, A. Mitsui, M. Yonemura, H. Iba and R. Kanno, Nat. Energy, 2016, 1, 16030.

5 W. Zhang, F. H. Richter, S. P. Culver, T. Leichtweiss, J. G. Lozano, C. Dietrich, P. G. Bruce, W. G. Zeier and J. Janek, ACS Appl. Mater. Interfaces, 2018, 10, 22226-22236.

6 R. Koerver, F. Walther, I. Aygün, J. Sann, C. Dietrich, W. G. Zeier and J. Janek, J. Mater. Chem. A, 2017, 5, 2275022760 .

7 W. Zhang, D. A. Weber, H. Weigand, T. Arlt, I. Manke, D. Schröder, R. Koerver, T. Leichtweiss, P. Hartmann, W. G. Zeier and J. Janek, ACS Appl. Mater. Interfaces, 2017, 9, 17835-17845.

8 R. Koerver, I. Aygün, T. Leichtweiß, C. Dietrich, W. Zhang, J. O. Binder, P. Hartmann, W. G. Zeier and J. Janek, Chem. Mater., 2017, 29, 5574-5582.

9 W. D. Richards, L. J. Miara, Y. Wang, J. C. Kim and G. Ceder, Chem. Mater., 2016, 28, 266-273.

10 L. Miara, A. Windmüller, C. L. Tsai, W. D. Richards, Q. Ma, S. Uhlenbruck, O. Guillon and G. Ceder, ACS Appl. Mater. Interfaces, 2016, 8, 26842-26850.

11 A. Sakuda, A. Hayashi and M. Tatsumisago, Chem. Mater., 2010, 22, 949-956.

12 F. Walther, R. Koerver, T. Fuchs, S. Ohno, J. Sann, M. Rohnke, W. G. Zeier and J. Janek, Chem. Mater., 2019, 31, 3745-3755.

13 Y. Tian, T. Shi, W. D. Richards, J. Li, J. C. Kim, S. H. Bo and G. Ceder, Energy Environ. Sci., 2017, 10, 1150-1166.

14 Y. Xiao, L. J. Miara, Y. Wang and G. Ceder, Joule, 2019, 3, 1252-1275.

15 N. Ohta, K. Takada, L. Zhang, R. Ma, M. Osada and T. Sasaki, Adv. Mater., 2006, 18, 2226-2229.

16 K. Takada, N. Ohta, L. Zhang, K. Fukuda, I. Sakaguchi, R. Ma, M. Osada and T. Sasaki, Solid State Ionics, 2008, 179, 1333-1337.
17 M. Tatsumisago, M. Nagao and A. Hayashi, J. Asian Ceram. Soc., 2013, 1, 17-25.

18 S. Ito, S. Fujiki, T. Yamada, Y. Aihara, Y. Park, T. Y. Kim, S. Baek, J. Lee, S. Doo and N. Machida, J. Power Sources, 2014, 248, 943-950.

19 A. O. Kondrakov, A. Schmidt, J. Xu, H. Geßwein, R. Mönig, P. Hartmann, H. Sommer, T. Brezesinski and J. Janek, J. Phys. Chem. C, 2017, 121, 3286-3294.

20 R. Weber, C. R. Fell, J. R. Dahn and S. Hy, J. Electrochem. Soc., 2017, 164, A2992-A2999.

21 F. P. McGrogan, T. Swamy, S. R. Bishop, E. Eggleton, L. Porz, X. Chen, Y. Chiang and K. J. Van Vliet, Adv. Energy Mater., 2017, 7, 1602011.

22 H. Tian and Y. Qi, J. Electrochem. Soc., 2017, 164, E3512E3521.

23 A. Neumann, S. Randau, K. Becker-Steinberger, T. Danner, S. Hein, Z. Ning, J. Marrow, F. H. Richter, J. Janek and A. Latz, ACS Appl. Mater. Interfaces, 2020, 12, 9277-9291.

24 R. Koerver, W. Zhang, L. De Biasi, S. Schweidler, A. O. Kondrakov, S. Kolling, T. Brezesinski, P. Hartmann, W. G. Zeier and J. Janek, Energy Environ. Sci., 2018, 11, 2142-2158.

25 T. Liu, Y. Zhang, R. Chen, S. X. Zhao, Y. Lin, C. W. Nan and Y. Shen, Electrochem. Commun., 2017, 79, 1-4.

26 T. Shi, Q. Tu, Y. Tian, Y. Xiao, L. J. Miara, O. Kononova and G. Ceder, Adv. Energy Mater., 2020, 10, 1902881.

27 Y. Zhang, Y. Tian, Y. Xiao, L. J. Miara, Y. Aihara, T. Tsujimura, T. Shi, M. C. Scott and G. Ceder, Adv. Energy Mater., 2020, 1903778.

28 Y. Mao, X. Wang, S. Xia, K. Zhang, C. Wei, S. Bak, Z. Shadike, X. Liu, Y. Yang, R. Xu, P. Pianetta, S. Ermon, E. Stavitski, K. Zhao, Z. Xu, F. Lin, X. Yang, E. Hu and Y. Liu, Adv. Funct. Mater., 2019, 29, 1900247.

29 K. Ishidzu, Y. Oka and T. Nakamura, Solid State Ionics, 2016, 288, 176-179.

30 J. Lee, A. Urban, X. Li, D. Su, G. Hautier and G. Ceder, Science, 2014, 343, 519-522.

31 F. Strauss, L. de Biasi, A. Kim, J. Hertle, S. Schweidler, J. Janek, P. Hartmann and T. Brezesinski, ACS Mater. Lett., 2020, 2, 84-88.

32 X. Ban, W. Zhang, N. Chen and C. Sun, J. Phys. Chem. C, 2018, 122, 9852-9858.

33 G. Tan, F. Wu, C. Zhan, J. Wang, D. Mu, J. Lu and K. Amine, Nano Lett., 2016, 16, 1960-1968.

34 S. Yu, S. Schmohl, Z. Liu, M. Hoffmeyer, N. Schön, F. Hausen, H. Tempel, H. Kungl, H. Wiemhöfer and R. Eichel, J. Mater. Chem. A, 2019, 7, 3882-3894. 